\title{
Severe TEN Management in a Patient with Schizophrenia
}

\author{
Fateme Tajic Rostami, ${ }^{1}$ Maryam Honardoost, ${ }^{2}$ and Zohreh Maghsoomi ${ }^{3,}$ \\ ${ }^{1}$ Faculty of Medicine, Iran University of Medical Sciences, Tehran, Iran \\ ${ }^{2}$ Endocrine Research Center, Institute of Endocrinology and Metabolism, Iran University of Medical Sciences, Tehran, Iran \\ ${ }^{3}$ Research Center for Prevention of Cardiovascular Disease, Institute of Endocrinology and Metabolism, Iran University of Medical Sciences, Tehran, Iran \\ "Corresponding author: Zohreh Maghsoomi, Research Center for Prevention of Cardiovascular Disease, Institute of Endocrinology and Metabolism, Iran University of Medical \\ Sciences, Tehran, Iran. E-mail: maghsoomi.z@iums.ac.ir
}

Received 2017 April 10; Accepted 2017 May 12.

\begin{abstract}
Background: Toxic epidermal necrolysis (TEN) is a rare and severe dermatologic reaction that could be life- threatening. Typically, it is related to or caused by various medicines that are most commonly antiepileptic. Secondary to epidermolysis, the skin and mucous membranes can also be involved. Severe burn injuries can be associated with acute kidney injury, sepsis, and respiratory dysfunction; however, regardless of severity, early diagnosis and proper treatment can improve the outcome.

Case Presentation: Here, we report a case of TEN that led to AKI, loss of conciseness, and respiratory failure in a patient with schizophrenia who had a good outcome despite the severity of the mentioned problems. Rigorous treatment included intensive fluid therapy, intravenous immunoglobulin, and wound coverage with a synthetic cover (silver) after gentle early debridement. Conclusions: This case report revealed that appropriate hydration, intravenous immunoglobulin, and high dose corticosteroid plus intensive wound care might be necessary to treat and improve outcomes in patients who suffer from severe TEN.
\end{abstract}

Keywords: Toxic Epidermal Necrolysis, Drug-Related Side Effects

\section{Background}

Stevens-Johnson syndrome and toxic epidermal necrolysis (SJS, TEN) are acute cutaneous reactions presented with macular lesion, followed by separation of the epidermis and dermis. The amount of mucocutaneous involvement may differentiate these two syndromes.

In Steven-Johnson syndrome (SJS), less than $10 \%$ of the body surface is involved, with $1 \%$ to $5 \%$ mortality. Surface involvement in TEN is more than $30 \%$ with $20 \%$ to $60 \%$ mortality, and $10 \%$ to $30 \%$ of the patient's skin are overlap of SJS/TEN. The annual rate of TEN is 0.4 to 1.3 cases per million population $(1,2)$. Several causes can lead to this reaction, the most important of which are medicines, but HLA differences in ethnic populations can lead to these syndromes with different medicines in different countries (3).

Prediction outcome of TEN can be estimated using SCORTEN (Score for Toxic Epidermal Necrolysis), which is included in BUN $>10 \mathrm{mmol} / \mathrm{L}$, bicarbonate $<20 \mathrm{mmol} / \mathrm{L}$, serum glucose $>14 \mathrm{mmol} / \mathrm{L}, \mathrm{HR}>120 \mathrm{bpm}$, and percentage of BSA with epidermal detachment $>10 \%$, and cancer, age $\geq 40$.The SCORTEN predicts mortality if 0 - 1 scores, $3.2 \%$; 2 scores, $12.1 \%$; 3 scores, $35.3 \%$; 4 scores, $58.3 \%$; and 5 or more scores, 90\% (4).

Despite the lack of treatment guidelines and high mortality, outcomes improved by immediately stopping the probable cause, wound care, and good coverage, intensive care unit admission, and several treatments such as corticosteroid, IVIG (2).

This case highlights that despite the poor prognosis estimated by SCORTEN, careful attention and administering IVIG might change the outcome.

\section{Case Presentation}

A 27- year- old Afghan male with decreased level of consciousness and progressive rash and bolus lesions was brought to the emergency room of Firooz Abadi hospital in the city of Shahre Rei by EMS. This lesion started on his face and lower extremity and spread to his entire body, affecting more than $70 \%$ of his total body surface area in 3 days. His face, eyes, and lips were swallowed and hardly opened. Because of genitalia involvement, catheterization was performed with difficulty. First, urine sample was decreased. Due to his respiratory distress, intubation was needed and it was hardly performed due to severe edema- 
tous of his lips and tongue. Vital signs showed HR: 140 bpm, BP: 100/60, and AT 39; however, other general examination results were normal except for the skin and mucosal membrane.

Bullous lesions with positive Nikolsky's sign and detached skin with necrotic area were seen over the body (head, neck, chest and abdomen, upper and lower extremities) (Figure 1).

He had a history of schizophrenia and was under treatment with some drugs which were changed three months ago, but he did not know the name of the drugs.

Laboratory findings were as follow: thrombocytopenia, anemia (Hb:11) with normal WBC, BUN: $35 \mathrm{mg} / \mathrm{dL}, \mathrm{Cr}$ : $1.5 \mathrm{mg} / \mathrm{dL}$, AST: $35 \mathrm{U} / \mathrm{L}$, ALT: $64 \mathrm{U} / \mathrm{L}$, ESR: 77 mm/h, CRP: 46.8 mg/L,Na: $145 \mathrm{mmol} / \mathrm{L}, \mathrm{K}: 4.9 \mathrm{mmol} / \mathrm{L}$, Albumin: $2.9 \mathrm{~g} / \mathrm{dL}$, LDH: 656 U/L, CPK: 690 U/L, Ca: 7.8 U/L, Mg: 1.9 mg/dL, ABG: $\mathrm{PH}: 7.3, \mathrm{HCO}_{3}: 33.5, \mathrm{PCO}_{2}: 60.4$ with $\mathrm{PAO} 2 / \mathrm{FIO}_{2}=195$.

Brain CT scan was normal, but chest x-ray showed a diffuse patchy alveolar pattern as ARDS.

Based on history, skin manifestation, and mount of involvement, the first diagnosis was TEN (toxic epidermal necrolysis). Predictor scores were calculated as follow: simplified acute physiology score (SAPS II) was 42 points with mortality percentage of $28.5 \%$, and SCORETEN was 3 points (BUN $>10 \mathrm{mmol} / \mathrm{L}$, heart rate $>120 / \mathrm{min}$, compromised body surface $>10 \%$ ), indicating a $35.3 \%$ mortality.

Our history, clinical examination, and lab data revealed sepsis, AKI, and ARDS. After ICU admission, a team of experts including an internist, a dermatologist, a wound care manager, a plastic surgeon, an immunologist, and an ophthalmologist managed him.

Aggressive IV fluid (normal saline) was initiated and continued to maintain urine output. IV PPI (pantoprazole $40 \mathrm{mg}$ IV daily) was used to prevent peptic ulcer, and IV antibiotic (meropenem + vancomycine with renal dose adjustment) was started. The dermatologist suggested to start corticosteroid pulse therapy and IVIG because of the skin involvement.

Daily debridement and dressing care with saline and silver-based bandage were done.

Other managements were supportive, topical drop, and restricted debridement for the eyes and libs, which were done daily.

Urine volume reached $950 \mathrm{cc}$ at the first day, and he was gradually conscious at the second day.

Methylprednisolone $1000 \mathrm{mg} / \mathrm{d}$ was started at early diagnosis for 3 consecutive days.

Intravenous immunoglobulin was prescribed $(2 \mathrm{~g} / \mathrm{kg}$ per day) from the second day of his admission and continued for three days. His body responded very well to our treatment and he was extubated after one week. His lesions gradually diminished. After extubation, he had complaints of odynophagia due to esophageal involvement that improved with a soft cold diet.

He was hospitalized for one month and was remarkably cured.

\section{Discussion}

TEN is a severe and serious mucocutaneous reaction that can lead to multiple organ failures $(2,4)$. The immune system has an important role in the diffuse desquamation of mucocutaneous and destruction of the skin (5). A wide spectrum of adverse drug reaction, from mild to severe, has been reported as erythema multiform to TEN (3). TEN manifestations are macular lesions in more than $10 \%$ of the body and positive Nikolsky's sign (6). Respiratory and genitourinary tract, mouth and esophagus, and eyes are often involved as mucositis. Systemic response is fever and manifestation of end organ damage. Lack of skin barrier and fever can lead to ongoing water loss, severe dehydration, and vasoconstriction that can result in AKI (7). Our patient had systemic complications such as AKI. Consciousness was decreased but improved after intensive hydration.

This reaction is rare, thus RCT cannot be performed easily. Despite no standard treatment, some articles report corticosteroid plus IVIG or plasmapheresis are useful for management of TEN (4). Conservative management is the most recommended approach. According to the past reports, we treated the patient with IVIG and corticosteroid.

The worst complication was infection (3). Thus, we advise the use of a broad spectrum of antibiotics, with corticosteroid for the first treatment (2). Also, IVIG prescription improved our patient outcome without any adverse effects. We did not know about causative medicines, but perhaps antipsychotics could have been responsible. Thus, at the time of his discharge, we wrote a letter to his physician and informed him of the patient's reaction to the medicine.

The prognostic score showed high mortality, however, wound care, IVIG plus high dose corticosteroid, and carefully conservative management could manage TEN. 

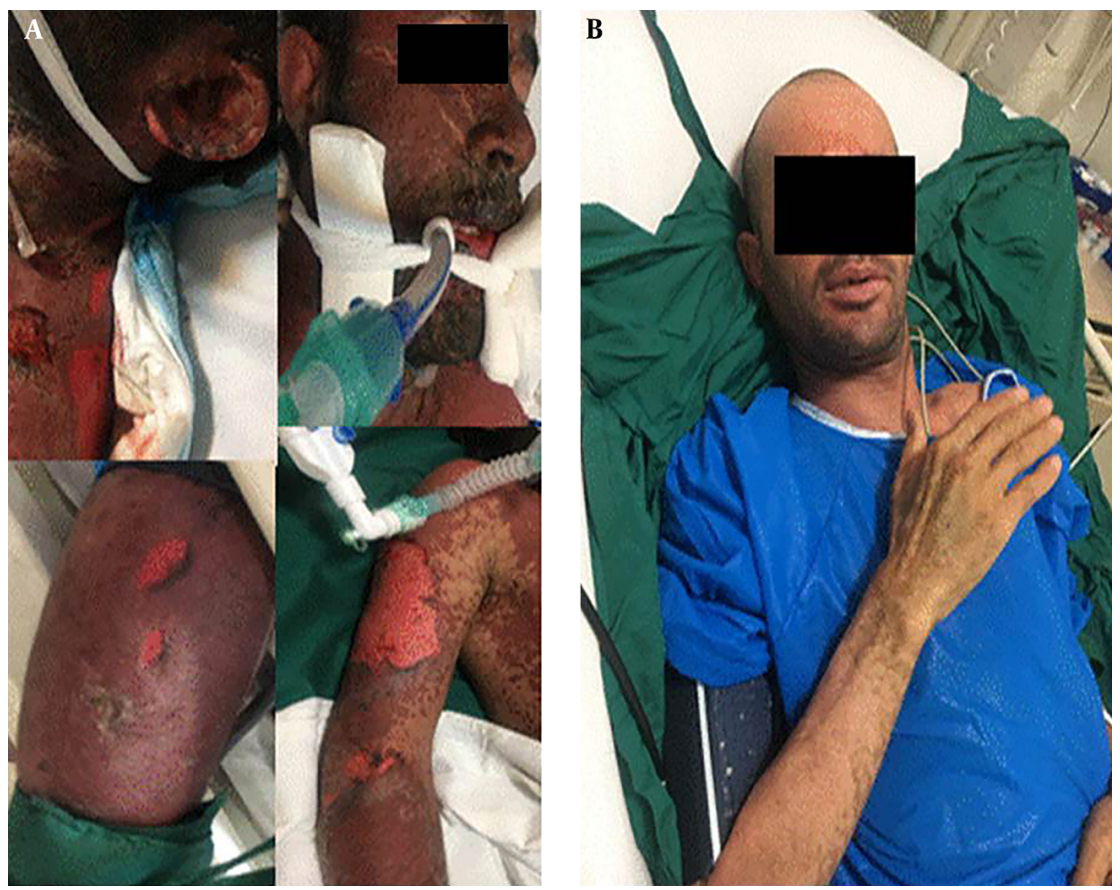

Figure 1. Detachment with necrosis skin and mucosal involvement in face, lower and upper extremities with positive Nikolsky's sign; A, before Treatment; B, skin rashes disappeared after one month of early treatment.

\section{References}

1. Wang YH, Chen CB, Tassaneeyakul W, Saito Y, Aihara M, Choon SE, et al. The Medication Risk of Stevens-Johnson Syndrome and Toxic Epidermal Necrolysis in Asians: The Major Drug Causality and Comparison to the USA FDA Label. Clin Pharmacol Ther. 2018. doi: 10.1002/cpt.1071. [PubMed: 29569740].

2. Klancir T, Nesek Adam V, Matolic M, Grizelj Stojcic E, Karaman Ilic M. Antibiotic-induced Toxic Epidermal Necrolysis - A Case Report. Acta Dermatovenerol Croat. 2017;25(1):72-6. [PubMed: 28511754].

3. Patel JB, Agrawal P, Soitawala S, Sattigeri BM. Amoxicillin induced toxic epidermal necrolysis (TEN): a case report. Int J Res Med Sci. 2017;3(4):1011-4.
4. Yamane Y, Matsukura S, Watanabe Y, Yamaguchi Y, Nakamura K, Kambara T, et al. Retrospective analysis of Stevens-Johnson syndrome and toxic epidermal necrolysis in 87 Japanese patients-Treatment and outcome. Allergol Int. 2016;65(1):74-81. doi: 10.1016/j.alit.2015.09.001. [PubMed: 26666483].

5. Purkayastha A. Toxic epidermal necrolysis: case report. Reactions. 2016;1614:151-13.

6. Rijal JP, Pompa T, Giri S, Bhatt VR. A case of toxic epidermal necrolysis caused by trimethoprim-sulfamethoxazole. BMJ Case Rep. 2014;2014. doi: 10.1136/bcr-2013-203163. [PubMed: 25008332]. [PubMed Central: PMC4091412].

7. Hu CH, Su ST, Chang NJ, Chung WH, Tian YC, Chuang SS, et al. Acute Kidney Injury in Toxic Epidermal Necrolysis Syndrome. 2013. 